\title{
A MODIFIED INITIAL CONDITION FOR CAUCHY'S EXISTENCE THEOREM
}

\section{J. PENDLETON BREWSTER ${ }^{1}$}

1. Introduction. For the differential system

$$
\begin{gathered}
f\left(x, y, u, u_{y}\right)=u_{x}, \\
u(0, y)=c(y),
\end{gathered}
$$

where $f, c, u$ are holomorphic functions of which $f, c$ are known and $u$ is unknown, Cauchy's theorem states the existence of a unique solution. The initial condition (1.2) effectively assigns the coefficients of the terms which involve $y$ alone.

Here is proved an existence theorem in which the initial condition assigns one of the $(n+1)$ coefficients of each order $n$. Corresponding to each non-negative integer $\boldsymbol{n}$ arbitrarily choose a pair of non-negative integers $\left(j_{n}, k_{n}\right)$ satisfying $j_{n}+k_{n}=n$. Select an infinite sequence of complex numbers $c_{n}$ subject only to the conditions

(i) that $f$ be holomorphic and $f_{u_{y}} \neq 0$ about $x=y=0, u=c_{0}, u_{y}=c$, where $c$ equals $c_{1}$ or satisfies $f\left(0,0, c_{0}, c\right)=c_{1}$ according as $j_{1}$ or $k_{1}$ is 0 ;

(ii) that

$$
h=\sum_{n=0}^{\infty} \frac{c_{n} x^{j_{n}} y^{k_{n}}}{j_{n} ! k_{n} !}
$$

have a pair of nonzero radii of convergence.

The initial condition to be employed is

$$
c_{n}=u_{j_{n} k_{n}}(0,0) \quad(n=0,1,2, \cdots),
$$

$u_{j k}$ denoting $u$ differentiated $j, k$ times with respect to $x, y$ respectively. A sequence $c_{n}$ satisfying (i) and (ii) is to be called an initial sequence.

The specific result proved here is

THEOREM 1.1. The determined differential system

$$
\begin{array}{cr}
f\left(x, y, u, u_{y}\right)=u_{x} & \left(f_{u_{y}} \neq 0\right), \\
c_{n}=u_{j_{n} k_{n}}(0,0) & (n=0,1,2, \cdots),
\end{array}
$$

where $f$ is a given holomorphic function and $c_{n}$ is an arbitrary initial sequence, has a unique holomorphic solution $u(x, y)$.

Received by the editors June 4, 1952.

1 The subject matter of this paper is taken from a doctoral thesis presented at Duke University. 
2. Equation pairs. An appeal to the implicit function theorem shows that with each holomorphic $f$ satisfying

$$
f_{u_{y}}\left[0,0, u(0,0), u_{y}(0,0)\right] \neq 0
$$

can be associated a partner $g$ to form an equation pair with the following properties:

The pair consists of the equations

$$
\begin{aligned}
& f\left(x, y, u, u_{y}\right)=u_{x}, \\
& g\left(x, y, u, u_{x}\right)=u_{y},
\end{aligned}
$$

where $f$ and $g$ are holomorphic. The numerical determination satisfies both equations, that is,

$$
\begin{aligned}
& f\left[0,0, u(0,0), u_{y}(0,0)\right]=u_{x}(0,0), \\
& g\left[0,0, u(0,0), u_{x}(0,0)\right]=u_{y}(0,0) .
\end{aligned}
$$

Under evaluation by (2.1) or by (2.2) and in particular for the values in (2.3), (2.4)

$$
f_{u_{y}} g_{u_{x}}=1 \text {. }
$$

3. The principal coefficients. Seek a solution

$$
u(x, y)=\sum_{j, k=0}^{\infty} \frac{u_{j k}(0,0)}{j ! k !} x^{j} y^{k}
$$

whose coefficients are found by differentiating (1.5) or directly from (1.6).

The coefficients $c_{n}$ are called parametric, the others, principal [2, p. 258]. The order of a coefficient is the order of the corresponding derivative.

Under all circumstances, the coefficient $u_{00}(0,0)$ is parametric and equals $c_{0}$.

Of the two coefficients, $u_{01}(0,0), u_{10}(0,0)$, one is principal and the other parametric. If $u_{01}(0,0)=c_{1}$, evaluation of $(2.1)$ gives

$$
f\left(0,0, c_{0}, c_{1}\right)=u_{10}(0,0) .
$$

To carry the illustration one step farther, suppose for the sake of definiteness

$$
c_{1}=u_{10}(0,0), \quad c_{2}=u_{11}(0,0) .
$$

Because of (2.3) the series

$$
f\left[0,0, c_{0}, u_{01}(0,0)\right]
$$


is convergent. So also are

$$
\begin{gathered}
f_{x}\left[0,0, c_{0}, u_{01}(0,0)\right], \quad f_{u}\left[0,0, c_{0}, u_{01}(0,0)\right], \\
f_{u_{y}}\left[0,0, c_{0}, u_{01}(0,0)\right]
\end{gathered}
$$

and the derived series of all orders for the same values. Similar statements are true for $g$ and its derivatives.

The principal coefficients of order two are given by

$$
\begin{aligned}
& f_{x}\left[0,0, c_{0}, u_{01}(0,0)\right]+f_{u}\left[0,0, c_{0}, u_{01}(0,0)\right] c_{1} \\
&+f_{u_{y}}\left[0,0, c_{0}, u_{01}(0,0)\right] c_{2}=u_{20}(0,0), \\
& g_{y}\left(0,0, c_{0}, c_{1}\right)+g_{u}\left(0,0, c_{0}, c_{1}\right) u_{01}(0,0)+g_{u_{x}}\left(0,0, c_{0}, c_{1}\right) c_{2}=u_{02}(0,0) .
\end{aligned}
$$

Both of these equal convergent infinite series in $c_{0}, c_{1}, c_{2}$ whose coefficients are polynomials in the coefficients of $f, g$.

Induction shows that the principal coefficients of order $n$ are expressed in terms of the parametric of order not exceeding $n$.

Apply all differentiations corresponding to the monomials $x^{j} y^{k}$ for $j+k=n-1$ and $j_{n} \leqq j$ to (2.1). Derivatives of order less than $n$ being ignored, this gives

$$
\left(j_{n}, k_{n}\right)<\left(j_{n}+1, k_{n}-1\right)<\cdots<(n-1,1)<(n, 0),
$$

a relation to be read from right to left " $u_{n 0}$ is expressed in terms of $u_{n-1,1}$, which in turn is expressed in terms of $u_{n-2,2}$, etc.

Similarly, apply all differentiations corresponding to the monomials $x^{i} y^{k}$ for $j+k=n-1$ and $k_{n} \leqq k$ to (2.2) with the result:

$$
\left(j_{n}, k_{n}\right)<\left(j_{n}-1, k_{n}+1\right)<\cdots<(1, n-1)<(0, n) .
$$

Every principal coefficient can consequently be found from $c_{0}, c_{1}, c_{2}, \cdots$ and the coefficients of $f, g$ by the operations,$+ \times$.

4. Specialization of system. It is convenient to replace the system $f, g$ in the foregoing discussion by $F, G$ with indeterminate coefficients. The series for its solution is called the indeterminate solution.

From the system so conceived the particular system for which Theorem 1.1 is to be proved arises by assigning to the indeterminates the values they have in the given $f, g$. The series for its solution will be called the tentative solution.

A second particular system, called dominant for the first, arises by assigning judiciously chosen values to the indeterminates. The solution of this system is called the dominant solution.

5. Reduction of initial sequence. Set

$$
u=v+h,
$$


where $h$ is the holomorphic function given by (1.3). The system (1.5), (1.6) is thereby carried [2, pp. 254, 260] into another system of the same form with all constants zero in the initial determination. Consequently, in proving Theorem 1.1 it may be assumed that $u_{j_{n} b_{n}}(0,0)=0$.

6. The dominant system. The system

$$
\frac{N}{(1-a x-b y-c U)\left(1-d U_{y}\right)}+\frac{1}{d}=U_{x},
$$

where $N, a, b, c, d$ are positive constants at our disposal, arises by specializing the indeterminate coefficients in (1.5). It is to be the dominant equation.

The partner for (6.1) is

$$
\frac{N}{(1-a x-b y-c U)\left(1-d U_{x}\right)}+\frac{1}{d}=U_{y} .
$$

Expansion of the left member of (6.1) gives

$$
N+d^{-1}+\sum_{1}^{\infty} N I a^{i} b^{j} c^{k} d^{l} x^{i} y^{j} U^{k} U_{y}^{l}=U_{x},
$$

$I$ being a positive integer variable with $i, j, k, l$, and expansion of (1.5) with $u_{00}(0,0)=u_{01}(0,0)=0$ gives

$$
f_{0000}+\sum_{1}^{\infty} \frac{f_{i j k l}}{i ! j ! k ! l !} x^{i} y^{j} u^{k} u_{y}^{l}=u_{x},
$$

the $f_{i j k l}$ being the derivatives of $f$ evaluated for all four arguments equal to zero.

From Cauchy's inequality

$$
\frac{\left|f_{i j k l}\right|}{i ! j ! k ! l !} \leqq M p^{-i} q^{-i} r^{-k} s^{-l}
$$

where $M, p, q, r, s$ are positive numbers. Subject $N, a, b, c, d$ to

$$
\begin{gathered}
M \leqq N, \quad p^{-1} \leqq a, \quad q^{-1} \leqq b, \quad r^{-1} \leqq c, \quad s^{-1} \leqq d, \\
\left|f_{0000}\right| \leqq N+d^{-1} .
\end{gathered}
$$

The coefficient in (6.3) then dominates its correspondent in (6.4).

The coefficients in the expansion of the left member of (6.2) are identical with those in (6.3). The coefficients of $g$ are dominated by (6.3) provided the left members in (6.5) are replaced by the greater of the two corresponding numbers for $f$ and $g$. 
7. Solution of dominant equation. A particular solution of (6.1) which is sufficient for the present purpose can be found by the usual method. Seek a solution which depends on $x, y$ only in the combination

$$
z=a x+b y
$$

and denote differentiation with respect to $z$ by an accent. Then

$$
U_{x}=a U^{\prime}, \quad U_{y}=b U^{\prime} .
$$

The equation becomes

$$
\frac{N}{1-z-c U}+\frac{1}{d}=(a+b) U^{\prime}-a b d U^{\prime 2} .
$$

Choose $U(0)=0$. The value $U^{\prime}(0)=w$, say, must then satisfy

$$
Q(w)=a b d w^{2}-(a+b) w+N+d^{-1}=0 .
$$

This equation has a simple root on

$$
0<w<\frac{a+b}{2 a b d}
$$

provided

$$
N+d^{-i}<\frac{(a+b)^{2}}{4 a b d} .
$$

Fix $b, d$. Since the right member increases with $a$, the last inequality can be satisfied.

Hence (7.1) has a root $U^{\prime}=P(z, U)$, where $P$ is holomorphic about $z=U=0$.

By Cauchy's theorem for an ordinary equation, (7.1) has a solution $U(z)$ holomorphic about $z=0$ and satisfying

$$
U(0)=0, \quad 0<U^{\prime}(0)=w .
$$

In order that the equal function $U(x, y)$ dominate $u(x, y)$, it is necessary and sufficient that the coefficients of higher order all be non-negative. That they are positive is shown by differentiating (7.1) and writing the result thus:

$$
\left(a+b-2 a b d U^{\prime}\right) U^{\prime \prime}=H\left(z, U, U^{\prime}\right),
$$

where $H$ is a series in $z, U, U^{\prime}$ with positive coefficients. Successive differentiation and evaluation give the desired result.

8. Convergence proof. The tentative solution is found by giving 
the indeterminate coefficients of $F, G$ their appropriate values and the parametric coefficients the value zero. The dominant solution is found by giving the indeterminate coefficients of $F, G$ the values they have in (6.1) and the parametric coefficients non-negative values. Because the calculation involves only the operations,$+ X$, each dominant coefficient is at least equal to the absolute value of the corresponding tentative coefficient.

9. Satisfaction proof. Write

$$
R=f-u_{x}, \quad S=g-u_{y} .
$$

Since the tentative solution $u(x, y)$ and its partial derivatives $u_{x}(x, y), u_{y}(x, y)$ are holomorphic, the substitution theorem for holomorphic functions [1, p. 51] shows that substitution of the series for $u(x, y)$ in (9.1) gives holomorphic functions $R(x, y), S(x, y)$. To prove that $u(x, y)$ satisfies the given system, it suffices to show that

$$
R_{j k}(0,0)=S_{j k}(0,0)=0 .
$$

These equations for $j=k=0$ amount to (2.3), (2.4). The proof proceeds by induction on $j+k$.

The crucial point is settled by the following remarks. From the implicit function theorem the systems

$$
R_{j k}=0, \quad j+k<n,
$$

and

$$
S_{j k}=0, \quad j+k<n,
$$

are equivalent in the sense that any values of the indeterminates

$$
u_{j k}, \quad j+k \leqq n,
$$

which satisfy one satisfy the other.

Now suppose in (9.3), (9.4), and all equations subsequently to be written the arguments evaluated for $x=y=0$. Consider the equation

$$
R_{j k}=\cdots+f_{u_{y}} u_{j, k+1}-u_{j+1, k}=0
$$

and its correspondent

$$
S_{j k}=\cdots+g_{u x} u_{j+1, k}-u_{j, k+1}=0 .
$$

Elimination of $u_{j, k+1}, u_{j+1, k}$ by use of (2.5) gives a relation among the derivatives of order less than $n-1$, which being implied by (9.3) must be implied by the equations of (9.3) not involving derivatives of order $n-1$, that is, by the system 


$$
R_{j k}=0,
$$$$
j+k<n-1 .
$$

Hence in the presence of (9.7) equations (9.5) and (9.6) are equivalent.

\section{REFERENCES}

1. W. F. Osgood, Lehrbuch der Funktionentheorie, vol. 2, part 1, Leipzig, 1929.

2. J. M. Thomas, Orderly differential systems, Duke Math. J. vol. 7 (1940) pp. 249-290.

DUKE UNIVERSITY

\section{RECENT INVESTIGATIONS OF THE SEPARATION OF LAPLACE'S EQUATION}

PARRY MOON AND DOMINA EBERLE SPENCER

Introduction. Two independent studies $[1 ; 2]$ have been made recently of separability conditions for the Laplace equation. Since the results of the two investigations are different, and even the meaning of "separability" is not identical, it seems pertinent to compare the two. We shall limit ourselves to euclidean 3-space and curvilinear coordinate systems $\left(u^{1}, u^{2}, u^{3}\right)$.

The meaning of "separability." According to [1], separation is of two kinds:

(a) If the assumption

$$
\phi=\prod_{i=1}^{3} U^{i}\left(u^{i}\right)
$$

permits the separation of the partial differential equation into three ordinary differential equations, the equation is said to be simply separable.

(b) If the assumption

$$
\phi=\frac{1}{R\left(u^{1}, u^{2}, u^{3}\right)} \prod_{i=1}^{3} U^{i}\left(u^{i}\right)
$$

permits the separation of the partial differential equation into three ordinary differential equations, and if $R \neq$ const., the equation is said to be $R$-separable.

These definitions seem to be in agreement with the usual meaning of the word "separability." Levinson, Bogert, and Redheffer [2],

Received by the editors June 11, 1952. 